\title{
Successful endoscopic identification of the bleeding source in the ventral dura of the cervical spine in a case of superficial siderosis
}

\author{
Yoshimichi Sato, MD, , ${ }^{1,2}$ Toshiki Endo, MD, PhD, ${ }^{1-3}$ Tomoo Inoue, MD, PhD, ${ }^{3}$ \\ Miki Fujimura, MD, PhD, ${ }^{1}$ and Teiji Tominaga, MD, PhD² \\ 1Department of Neurosurgery, Kohnan Hospital, Sendai; '2Department of Neurosurgery, Tohoku University Graduate School of \\ Medicine, Sendai; and ${ }^{3}$ Department of Neurosurgery, Sendai Medical Center, Sendai, Japan
}

\begin{abstract}
The authors report on the case of a 65-year-old man suffering progressive gait disturbance and hearing impairment due to superficial siderosis (SS). According to the literature, repeated hemorrhage into the subarachnoid space causes SS; however, the bleeding source remains unknown in half of SS patients. In the presented case, preoperative MRI revealed a fluid-filled intraspinal cavity extending from $\mathrm{C} 2$ to $\mathrm{T} 8$ with a dural defect at the ventral $\mathrm{C} 7$ level. During surgery, the dural defect was seen to connect to the intraspinal cavity filled with xanthochromic fluid. Importantly, endoscopic observation verified that the rupture of fragile bridging veins in the cavity was the definite bleeding source. Postoperative MRI confirmed disappearance of the intraspinal cavity, and the patient's symptoms gradually improved. The use of endoscopy helped to establish the diagnosis and led to definite treatment. Fragile bridging veins in the fluid-filled interdural layers were novelly verified as a bleeding source in SS. Recognizing this phenomenon is important since it can establish closure of the dural defect as a definite treatment in SS with an intraspinal cavity.
\end{abstract}

https://thejns.org/doi/abs/10.3171/2019.12.SPINE191102

KEYWORDS superficial siderosis; endoscopy; bleeding source; dural defect; cervical

S UPERFICIAL siderosis (SS) of the central nervous system (CNS) is a rare disease that is caused by repeated hemorrhage into the subarachnoid space. ${ }^{16}$ Hemosiderin deposition in subpial layers of the brain and spinal cord can cause neurological deficits. ${ }^{15}$ According to previous reports, the etiology of SS includes CNS tumors, arteriovenous malformations, and a history of head and neck trauma. ${ }^{9,23,27}$ Surgical treatments for intracranial and spinal pathologies per se can cause SS..$^{19,29}$ However, determining a definite cause of the bleeding is challenging. In half of SS cases, bleeding sources remain unknown. ${ }^{16}$

Here, we report a unique case of SS, in which preoperative MRI indicated a defect in the ventral C7 dura mater as well as an intraspinal cavity extending from C2 to T8. During surgery, an endoscope was inserted through the dural defect and revealed fragile bridging veins running in the interdural space as a bleeding source causing SS. A dural defect and fluid-filled intraspinal cavity have been reported in SS cases, ${ }^{1,7,14,16,28}$ however, the underlying pathology has not yet been confirmed. ${ }^{2}$ Xanthochromic fluid may have leaked through the dural defect into the subarachnoid space, leading to SS.

This, to our knowledge, is the first report in which an endoscope was used to directly observe microbleedings from fragile bridging veins in the interdural space. Endoscopy has been used to verify this important phenomenon as a definite bleeding source in SS.

\section{Case Report}

History and Examination

A 65-year-old man suffering progressive gait disturbance and hearing impairments was referred to our hospital. The patient did not have positional headache. Brain T2*-weighted MRI performed on admission revealed lowintensity rims throughout the brainstem, cerebellum, and sylvian and interhemispheric cisterns (Fig. 1), which were consistent with hemosiderin deposits. No tumors or vascular pathologies were evident on brain and spinal cord MRI. Rather, sagittal T2-weighted MRI demonstrated a 

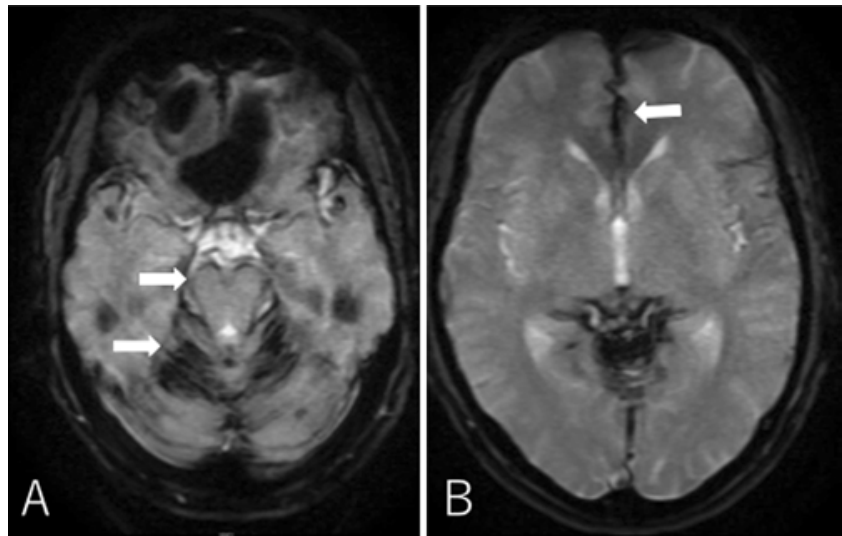

FIG. 1. Preoperative axial T2*-weighted MR images showing hemosiderin deposition on the brainstem and cerebellum (arrows, $\mathbf{A}$ ) as well as in the interhemispheric fissure (arrow, B).

fluid-filled cavity from $\mathrm{C} 2$ to $\mathrm{T} 8$ in the ventral side of the cervicothoracic spine (Fig. 2A). Heavily T2-weighted sagittal and axial images indicated a dural defect at the right ventral C7-T1 level (Fig. 2B and C). Surgery was offered to explore and close the dural defect with the intention to stop the bleeding to allow recovery from the SS.

\section{Operation}

The patient was positioned prone. Following $\mathrm{C} 7$ and T1 laminectomy and durotomy, the dural defect was revealed as had been demonstrated on preoperative MRI (Fig. 3A). The dural defect was connected to the fluidfilled cavity, where bloody fluid pooled. A 3-mm angled endoscope $\left(30^{\circ}\right)$ was inserted through the defect into the cavity (Fig. 3B). Endoscopic observation provided two important findings: the cavity was formed between the dural layers, and bridging veins running between the dural layers with trabeculae were fragile and continued to bleed (Fig. 3C). As we confirmed the interdural fragile bridging veins as the bleeding source of SS, we sutured the dural defect and covered it with polyglycolic acid felt and fibrin glue to complete the surgery (Fig. 3D and E and Video 1).

VIDEO 1. After $\mathrm{C} 7$ and $\mathrm{T} 1$ laminectomy, the dura and arachnoid were cut. On the right side and ventral to the spinal cord, the dural defect was identified using a microscope. Bloody fluid was suctioned via a syringe, suggesting that the bleeding occurred in the intraspinal cavity. Then, an endoscope was inserted through the defect into the cavity. Between the dural layers, bridging veins and trabeculae were noted. Bleeding continued from the bridging vein. The dura was sutured and covered with polyglycolic acid felt and fibrin glue. Copyright Toshiki Endo. Published with permission. Click here to view.

\section{Postoperative Course}

Two days after the operation, postoperative MRI confirmed that the fluid-filled intraspinal cavity had shrunk (Fig. 4A). Two weeks after surgery, the patient was discharged home. His symptoms had gradually improved by the 1-year outpatient clinic follow-up. MRI confirmed disappearance of the intraspinal cavity (Fig. 4B).
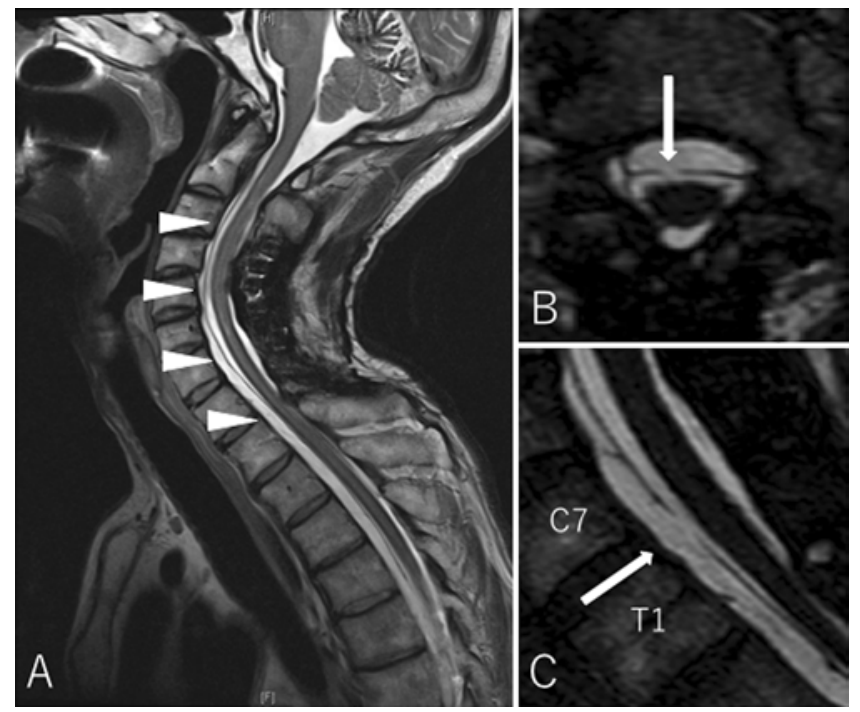

FIG. 2. Preoperative cervicothoracic sagittal T2-weighted MR image (A) showing a fluid-filled cavity ventrally from C2 to T8 (arrowheads). Preoperative cervicothoracic axial (B) and sagittal (C) heavily T2-weighted MR images clearly indicating a dural defect in the right ventral dura at C7-T1 (arrows).

\section{Discussion}

Patients with SS frequently have a dural defect and a fluid-filled intraspinal cavity. ${ }^{14}$ Dural defects usually occur spontaneously; however, trauma and degenerative disc disease or an osteophyte can also be associated. ${ }^{6,17,22,26}$

Accurate diagnosis of SS requires identification of the dural defect in the intraspinal cavity, and CT myelography has been reported to be useful in locating high-flow cerebrospinal fluid (CSF) leakage through the dural defect. ${ }^{14,20}$ Recently, high-resolution constructive interference in steady state (CISS) MRI was applied and detected a dural defect in SS. ${ }^{11}$ CISS MRI demonstrated high soft-tissue contrast ${ }^{10}$ which made it possible to detect a dural defect and thus was used in the current case.

To date, the relationship between the fluid-filled intraspinal cavity and chronic bleeding has not been fully elucidated. However, several hypotheses have been proposed. One theory is that continuous CSF leakage may lead to intradural hypotension and engorgement of epidural veins. Subsequent microtrauma to the epidural veins may cause SS since epidural blood may circulate into the subarachnoid space. ${ }^{5}$ Chronic intradural bleeding may also result from superior cerebellar bridging veins that are stretched because of brain sagging due to intracranial hypotension. ${ }^{16,24,25}$ Furthermore, it is known that intracranial hypotension leads to enlarging intraspinal veins. ${ }^{4}$ Continuous CSF leakage and venous hypertension may promote microtrauma of the enlarged fragile vessels adjacent to the dural defect. ${ }^{18}$

Recently, Hosokawa et al. postulated another possible mechanism of SS-dissection of the dura mater. ${ }^{11}$ After a dural tear, CSF flows into the space between the dural layers to make an intraspinal intradural cavity. This is in line with the concept of "duropathy" that Kumar has proposed..$^{13}$ Our surgical findings novelly confirmed fragile 


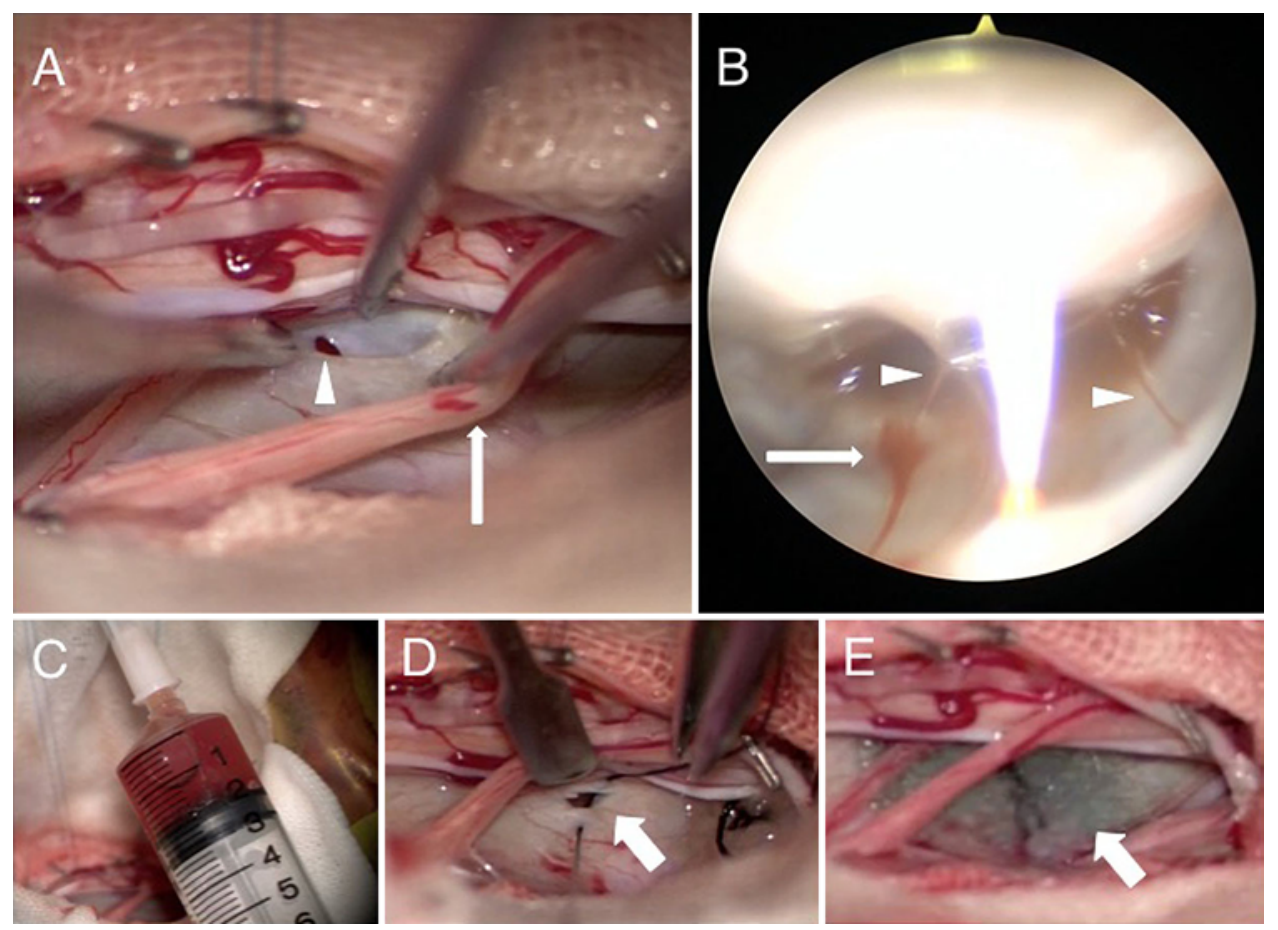

FIG. 3. Intraoperative photographs. A: Dural defect (arrowhead) in the ventral dura, just as preoperative MRI had indicated. Arrow indicates the right T1 dorsal nerve root. B: Endoscopic observation revealed fragile bridging veins (arrowheads) inside the fluid-filled cavity. Note that there was bleeding from one of the veins (arrow). C: Bloody fluid was suctioned from inside the cavity. D and E: The dural defect was sutured and covered with polyglycolic acid felt and fibrin glue (arrows). Figure is available in color online only.

bridging veins running in the interdural space as a bleeding source, which supports Kumar's new hypothesis of duropathy as a plausible mechanism of SS.

Endoscopy has evolved as a useful adjunct in spinal surgery. ${ }^{8,12}$ In one of the first clinical applications of its type, we used flexible endoscopy in the surgical treatment of arachnoid cysts that extended over multiple vertebral levels in a craniocaudal direction. ${ }^{8}$ Despite this extension, an endoscope was moved longitudinally along the spinal cord, reaching both cranial and caudal edges of the cyst from a one-level laminectomy. We have also utilized an angled endoscope to observe via posterolateral approaches the ventral surface of the spinal cord in cases of spinal arteriovenous malformations. ${ }^{21}$ Endoscopy provides surgical views different from those of microscopy, obviating the need to manipulate or rotate the spinal cord. Overall, endoscopy is minimally invasive and prevents complications, thereby reducing postoperative pain and hospital stays. ${ }^{3}$

In this study, a rigid angled endoscope was inserted into the intraspinal cavity through a dural tear to locate the dural defect in SS patients. ${ }^{1}$ The endoscope reached inside the interdural space, providing detailed information on the fragile bridging veins. These features of endoscopy helped us to detect important surgical findings that would be otherwise overlooked.

Several studies have recommended surgical closure of the dural defect in SS.7,11,17 In our case, after surgical closure of the dural defect, stabilization of the neurological symptoms occurred, and imaging evidence confirmed resolution of the fluid-filled cavity. Efforts should be made to find a dural defect and close it surgically since the continued exposure of neural tissues to neurotoxic hemosiderin deposits can result in progressive neurological disturbance?
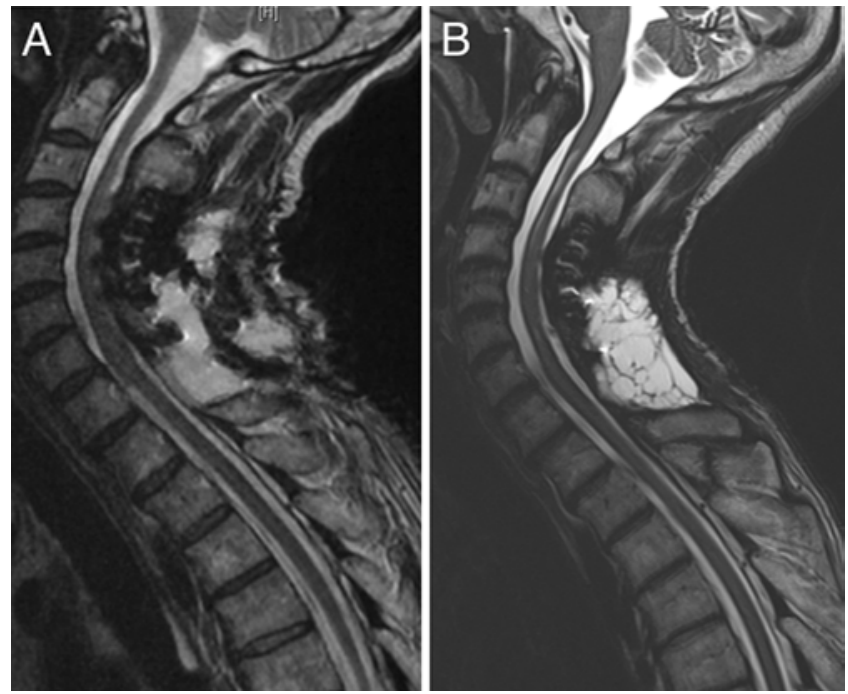

FIG. 4. Cervicothoracic sagittal T2-weighted MR images obtained 2 days (A) and 1 year (B) after surgery, confirming disappearance of the fluid-filled intraspinal cavity. 


\section{Conclusions}

This is the first report on the assisted use of endoscopy to identify a direct bleeding source of SS in the fluid-filled interdural cavity. We propose fragile bridging veins inside the cavity as a definite etiology of SS.

\section{Acknowledgments}

We thank Enago for the English-language review.

\section{References}

1. Arishima H, Higashino Y, Yamada S, Akazawa A, Arai H, Tsunetoshi K, et al: Spinal endoscopy combined with selective CT myelography for dural closure of the spinal dural defect with superficial siderosis: technical note. J Neurosurg Spine 28:96-102, 2018

2. Ball BG, Luetmer PH, Giannini C, Mokri B, Kumar N, Piepgras DG: Ventral "spinal epidural meningeal cysts"-not epidural and not cysts? Case series and review of the literature. Neurosurgery 70:320-328, 2012

3. Baron EM, Levene HB, Heller JE, Jallo JI, Loftus CM, Dominique DA: Neuroendoscopy for spinal disorders: a brief review. Neurosurg Focus 19(6):E5, 2005

4. Burtis MT, Ulmer JL, Miller GA, Barboli AC, Koss SA, Brown WD: Intradural spinal vein enlargement in craniospinal hypotension. AJNR Am J Neuroradiol 26:34-38, 2005

5. Cheng CY, Chen MH, Wang SJ, Lin KP: A proposed mechanism of superficial siderosis supported by surgical and neuroimaging findings. Med Hypotheses 76:823-826, 2011

6. Deluca GC, Boes CJ, Krueger BR, Mokri B, Kumar N: Ventral intraspinal fluid-filled collection secondary to CSF leak presenting as bibrachial amyotrophy. Neurology 76:14391440, 2011

7. Egawa S, Yoshii T, Sakaki K, Inose H, Kato T, Kawabata S, et al: Dural closure for the treatment of superficial siderosis. J Neurosurg Spine 18:388-393, 2013

8. Endo T, Takahashi T, Jokura H, Tominaga T: Surgical treatment of spinal intradural arachnoid cysts using endoscopy. J Neurosurg Spine 12:641-646, 2010

9. Fearnley JM, Stevens JM, Rudge P: Superficial siderosis of the central nervous system. Brain 118:1051-1066, 1995

10. Hingwala D, Chatterjee S, Kesavadas C, Thomas B, Kapilamoorthy TR: Applications of 3D CISS sequence for problem solving in neuroimaging. Indian J Radiol Imaging 21:90-97, 2011

11. Hosokawa M, Murata KY, Hironishi M, Koh J, Nishioka K, Nakao N, et al: Superficial siderosis associated with duplicated dura mater detected by CISS reverse MRI. J Neurol Sci 392:38-43, 2018

12. Ito A, Endo T, Inoue T, Endo H, Sato K, Tominaga T: Use of indocyanine green fluorescence endoscopy to treat concurrent perimedullary and dural arteriovenous fistulas in the cervical spine. World Neurosurg 101:814.e1-814.e6, 2017

13. Kumar N: Beyond superficial siderosis: introducing "duropathies." Neurology 78:1992-1999, 2012

14. Kumar N: Neuroimaging in superficial siderosis: an in-depth look. AJNR Am J Neuroradiol 31:5-14, 2010

15. Kumar N: Superficial siderosis: associations and therapeutic implications. Arch Neurol 64:491-496, 2007

16. Kumar N, Cohen-Gadol AA, Wright RA, Miller GM, Piepgras DG, Ahlskog JE: Superficial siderosis. Neurology 66:1144-1152, 2006

17. Kumar N, Lane JI, Piepgras DG: Superficial siderosis: sealing the defect. Neurology 72:671-673, 2009

18. Kumar N, McKeon A, Rabinstein AA, Kalina P, Ahlskog JE, Mokri B: Superficial siderosis and CSF hypovolemia: the defect (dural) in the link. Neurology 69:925-926, 2007
19. Kumar R, Jacob JT, Welker KM, Cutrer FM, Link MJ, Atkinson JL, et al: Superficial siderosis of the central nervous system associated with incomplete dural closure following posterior fossa surgery: report of 3 cases. J Neurosurg 123:1326-1330, 2015

20. Luetmer PH, Mokri B: Dynamic CT myelography: a technique for localizing high-flow spinal cerebrospinal fluid leaks. AJNR Am J Neuroradiol 24:1711-1714, 2003

21. Mansour A, Endo T, Inoue T, Sato K, Endo H, Fujimura M, et al: Clipping of an anterior spinal artery aneurysm using an endoscopic fluorescence imaging system for craniocervical junction epidural arteriovenous fistula: technical note. J Neurosurg Spine 31:279-284, 2019

22. Payer M, Sottas C, Bonvin C: Superficial siderosis of the central nervous system: secondary progression despite successful surgical treatment, mimicking amyotrophic lateral sclerosis. Case report and review. Acta Neurochir (Wien) 152:1411-1416, 2010

23. Schievink WI, Apostolides PJ, Spetzler RF: Surgical treatment of superficial siderosis associated with a spinal arteriovenous malformation. Case report. J Neurosurg 89:1029_ 1031, 1998

24. Schievink WI, Maya MM, Nuño M: Chronic cerebellar hemorrhage in spontaneous intracranial hypotension: association with ventral spinal cerebrospinal fluid leaks: clinical article. J Neurosurg Spine 15:433-440, 2011

25. Schievink WI, Wasserstein P, Maya MM: Intraspinal hemorrhage in spontaneous intracranial hypotension: link to superficial siderosis? Report of 2 cases. J Neurosurg Spine 24:454-456, 2016

26. Shih P, Yang BP, Batjer HH, Liu JC: Surgical management of superficial siderosis. Spine J 9:e16-e19, 2009

27. Tosaka M, Sato K, Amanuma M, Higuchi T, Arai M, Aishima K, et al: Superficial siderosis of the central nervous system caused by hemorrhagic intraventricular craniopharyngioma: case report and literature review. Neurol Med Chir (Tokyo) 55:89-94, 2015

28. Wilden JA, Kumar N, Murali HR, Lindell EP, Davis DH: Unusual neuroimaging in superficial siderosis. Neurology 65:489, 2005

29. Yokosuka J, Takai K, Komori T, Taniguchi M: Superficial siderosis: bleeding from the bone marrow after laminectomy for spinal tumor removal. J Neurosurg Spine 21:905-908, 2014

\section{Disclosures}

The authors report no conflict of interest concerning the materials or methods used in this study or the findings specified in this paper.

\section{Author Contributions}

Conception and design: Endo, Tominaga. Acquisition of data: Endo, Sato, Inoue. Analysis and interpretation of data: Endo, Fujimura. Drafting the article: Endo, Sato. Critically revising the article: Inoue, Fujimura, Tominaga. Reviewed submitted version of manuscript: all authors. Approved the final version of the manuscript on behalf of all authors: Endo. Study supervision: Fujimura, Tominaga.

\section{Supplemental Information \\ Videos \\ Video 1. https://vimeo.com/383743440.}

\section{Correspondence}

Toshiki Endo: Tohoku University Graduate School of Medicine, Sendai, Japan.endo@nsg.med.tohoku.ac.jp. 